\title{
Marruecos y Túnez: Un escenario de conflicto para los principios de occidente en el marco de una democracia impuesta.*
}

\section{Morocco and Tunisia: A conflict scenario for the western principles within the framework of a imposed democracy}

Karol Johana Mora Gómez**

\section{Resumen}

Esta investigación se realiza desde una metodología cualitativa descriptiva, la cual permite realizar un análisis crítico frente a la consolidación del Estado que marcó a África, especialmente a los Estados de Marruecos y Túnez, así como su evolución hacia la democracia. De este modo, se tendrán en cuenta los determinantes sociales, económicos, culturales y políticos que delimitaron su actual sistema político. Adicionalmente, esta investigación está apoyada en conceptos clave como democracia, geopolítica y Estados híbridos. Se considera que estos conceptos son herramientas de análisis que permiten la comprensión de la siguiente pregunta de investigación: ¿Cuáles son las implicaciones políticas de una occidentalización en el mundo árabe y su reconfiguración jerárquica subcontinental en el

1 Artículo de Revisión.

2 Karol Johana Mora Gómez. Contacto: kmora@academia.usbbog.edu.co 
balance de poder? La Investigación se centrará en la primera década del siglo xxi y las últimas décadas del siglo xx, este periodo fue seleccionado ya que marca un punto crucial para la transformación del sistema político de la región y, en especial, de Marruecos y Túnez; Estados que se entrarán a analizar.

\section{Palabras claves}

Balance de poder, transición de poder, democracia, poder real.

\section{Abstract}

This research will be carried out using a descriptive qualitative methodology which allows a critical analysis to be carried out regarding the consolidation of the State that marked Africa, especially the States of Morocco and Tunisia, as well as its evolution towards democracy, in this way they will be taken into account the social, economic, cultural and political determinants that delimited their current political system.

Additionally, this research is supported by key concepts such as democracy, geopolitics, and hybrid states. Believing that these concepts complement the research to the point that allows its understanding by reinforcing the central question. What are the political implications of a Westernization in the Arab World and its subcontinental hierarchical reconfiguration in the balance of power? The Research will focus on a period of ten years from the beginning of the intifada, this period was selected as it marks a crucial point for the transformation of the political system of the region and especially of the States that will be analyzed.

\section{Key Words}

Balance of power, transition of power, democracy, real power. 


\section{Introducción}

Altable J. (2000) asevera que el conflicto de Oriente Próximo es el más llamativo, complejo y prolongado de todos los que reclaman la atención de la opinión pública occidental. Judíos y árabes son los forjadores de grandes culturas, a su vez, emparentadas entre sí, que han definido en gran medida la manera europea de entender la vida.

El término "tercer mundo" fue adoptado durante el siglo xx para describir al grupo de los No Alineados y que luego se entendió como un término especialmente desarrollo para los países en vías de desarrollo y mucho menos desarrollados en comparación, los denominados subdesarrollados.

África ha sufrido un fenómeno de "transculturación", adoptando los valores y principios de Occidente; desde la época de la colonia se entienden las relaciones de centro-periferias, donde el primer mundo mediante dinámicas de dominación logra someter y establecer políticas de pobreza e implantar un desarrollo limitado para las regiones en las que se estableció como el principal extractor de recursos.

Del mismo modo, Grovogui S. N. (2011) asegura que existen dos problemáticas que permea la realidad del tercer mundo: en primer lugar, está la organización orgánica que busca librarse y legitimar su Estado-nación, y, en segundo lugar, plantea que la soberanía postcolonial constituye una desviación histórica del sistema westfaliano, tanto en lo relativo a la ficción jurídica como a la realidad empírica. Para lograr demostrar lo anterior, Grovogui, S. N. (2011) hace uso de la soberanía histórica bajo la influencia de la hegemonía occidentales, especialmente de los europeos.

Ahora bien, a manera de introducción, Marruecos y Túnez se encuentran ubicados en el Magreb, norte de África, y luego del 
acuerdo del Sykes-Picot (1916) se convierten en colonias francesas. Evidentemente por su cercanía y frontera con el mediterráneo, el intercambio cultural se facilita, lo cual conlleva a una occidentalización mayor, que podría sustentar el éxito o estabilidad de dichos Estados actualmente, así como su proximidad con Europa en materia idiosincrática y económica. Por otro lado, el éxito de la democracia se entiende, entonces, como la protección de los derechos humanos, civiles y políticos. Sin embargo, la protección y promulgación de los derechos civiles y políticos en países árabes discrimina prácticas tradicionales culturales de los pueblos árabes y musulmanes; pero el ejercicio de los mismos implica el triunfo e imposición de una normalidad subjetiva de un sistema capitalista, de los derechos civiles y políticos, de una legalidad y legitimidad del sufragio universal, una división de poderes, un andamiaje institucional óptimo, un desarrollo humano, entre otros acontecimientos "normales" en la cultura occidental, que permiten una ola de democratización y fortalecen los pilares de la democracia bajo un interés general y responsabilidad estatal y global.

Sin embargo, para poder responder adecuadamente a la pregunta ¿́cuáles son las implicaciones políticas de una occidentalización en el mundo árabe y su reconfiguración jerárquica subcontinental en el balance de poder?, especialmente en Marruecos y Túnez, a través de la teoría de transición de poder con un enfoque racionalista, es menester tener presente el sistema y régimen político de cada uno de los países a comparar, ya que a partir de allí se busca identificar las implicaciones políticas de una occidentalización en el mundo árabe, especialmente en Marruecos y Túnez, en el marco de una democracia impuesta por Occidente.

En este caso, el sistema político de Marruecos es una monarquía constitucional; mientras que en el caso de Túnez es una república parlamentaria. Este último cuenta con una particularidad actualmente y es que es laico. Pero, aun si su población es en su ma- 
yoría musulmana y constitucionalmente se reconoce al islam como su religión oficial, se respeta la libertad de culto; por otro lado, la población marroquí es un 99,4 \% musulmana sunní y cuenta con movimientos salafistas.

Esta investigación se realiza desde una metodología cualitativa descriptiva que permite realizar un análisis crítico de la consolidación de los Estados del tercer mundo, especialmente los africanos y su evolución hacia la democracia, así como los impactos sociales, económicos, culturales y políticos en su sistema político; además de realizar una revisión bibliográfica, se pretende desarrollar un caso comparado entre Marruecos y Túnez frente a las olas de democratización que han atravesado luego de la intifada. Sin embargo, no solo se hizo uso de documentación estrictamente académica para la construcción del documento, sino también de documentos literarios, artículos de opinión independientes y periodísticos, lo que permitió realizar un análisis del manejo de prensa y la respuesta del público. Del mismo modo y siguiendo con esta lógica, se hizo uso de documentales y apoyos fílmicos que visualicen la complejidad de la sociedad en oriente próximo, también conocido como el mundo árabe.

Para el desarrollo de la investigación se plantea como objetivo general identificar el motor que determina la imposición jerárquica en el balance de poder subcontinental a la luz de la teoría de la transición de poder desde un enfoque racionalista.

Adicionalmente, se plantean como objetivos específicos, en primer lugar, interpretar la consolidación estatal tradicional wesfaliano y su transición durante el siglo Xxi hacia los "autoritarismos competitivos"; en segundo lugar, la posibilidad de reconocer cuáles son los cambios dramáticos en el balance de poder regional e internacional que posibilitan las olas de cambio de régimen. Finalmente, se pretende analizar los conflictos político-administrativos que sostienen 
la jerarquía global y su influencia en la política exterior de Marruecos y Túnez, lo cual impactará directamente en su sistema político, régimen político y sus olas de democratización.

Marruecos y Túnez se encuentran ubicados en el Magreb, norte de África y luego del acuerdo de Sykes-Picot (1916) se convierten en colonias francesas, evidentemente por su cercanía y frontera con el mediterráneo el intercambio cultural se facilita, lo cual conlleva a que se diera una occidentalización mayor, esto sustenta su éxito en materia comercial así como una estabilidad al interior de los Estados, de modo que gracias a la "Euráfrica" -se refiere específicamente a la complementariedad entre Europa y África durante el periodo colonial- se mantiene una relación constante en materia económica e idiosincrática, lo cual permitió que la "misión civilizadora" por parte de Francia se desarrollara e incluso se cumpliera en el artículo 86 de la nueva constitución (1958), donde si un Estado se hacía independiente, ya no podría pertenecer a la Comunidad Francesa (Communauté Française), consolidada en su primer artículo.

Por otro lado, la ley del 30 de abril de 1946 planteaba que la ayuda francesa, debía estar concentrada en los territorios previamente administrados por Francia como colonias, pero estas "donaciones" o ayudas económicas contaban con grandes medidas restrictivas, puesto que solo las empresas francesas o pertenecientes a los países beneficiarios podían llevar a cabo actividades financiadas por el denominado Fonds d'Aide et de Coopération (FAC) transformando así a la "cooperación jerárquica” vertical según la teoría neoclásica de desarrollo ya que continúa con una lógica colonial tradicional o sociedad tradicional, Rostow (1961) en las etapas de desarrollo plantea tres nociones constitutivas básicas donde las sociedades tradicionales realizan transición a una modernización, dicho modelo es aplicable para los países menos desarrollados ya que deja de lado el crecimiento económico y se centra en el enriquecimiento político. 
Ahora bien, Gunitsky (2017), en su obra Aftershocks: Great Powers and Domestic Reforms in the Twentieth Century, propone tres mecanismos causales por los cuales se desarrollan las olas de cambio y sostiene la hipótesis de que: los cambios dramáticos en el balance de poder internacional son los que finalmente posibilitan las olas de cambio de régimen; esto permite que los Estados transiten hacia un autoritarismo competitivo, cambiando a su vez las lógicas de la arena internacional.

Gunitsky (2017) propone que el primer mecanismo es la coerción hegemónica, en la cual existe un desfase en el balance de poder regional, donde se evidencia una latente invasión y ocupación por parte de las potencias hacia las periferias del sistema internacional, lo cual impulsa a una carrera armamentística por parte de las periferias que tiene como único fin evitar la imposición y coerción de los centros. El segundo mecanismo que plantea Gunitsky (2017) es la provisión de incentivos, está alude a la capacidad que tiene el hegemónico ascendiente para expandir sus redes de intercambio, posibilitando una cooperación entre iguales o de interdependencia de manera horizontal y ya no vertical, como se mantenía en un principio bajo una visión colonialista. La cooperación sur-sur se puede dar de manera bilateral y multilateral en el caso de este último que la interacción entre los actores es mayor y se da no necesariamente entre instituciones gubernamentales (de Estado a Estado). En las dinámicas contemporáneas las relaciones de cooperación se pueden dar entre ONG, empresas, sindicatos y otros involucrando, impactando directamente en la población civil. Las interacciones entre los actores son las que finalmente posibilitan un cambio en el sistema y de régimen, lo mismo pasa con las demandas, las oportunidades de mercado, el comportamiento, las necesidades y el lenguaje de la población. Por último, el tercer mecanismo planteado por Gunitsky (2017) es la emulación, en este caso se trata del impacto hegemónico que revela la efectividad del régimen y contribuye a su legitimación. 
Por otro lado, no todas las olas tienen éxito, como en el caso de Túnez, ya que el éxito de las mismas depende del impacto hegemónico que tenga al interior de los Estados; este debe contribuir a la consolidación del andamiaje institucional tal como se ha evidenciado después de la Primavera árabe. Los países del mundo árabe y Medio Oriente han sufrido turbulencias en la consolidación de sus instituciones al interior de los gobiernos, lo cual imposibilita, a su vez, la proyección efectiva de su política exterior y su generación de redes, que en pocas palabras se traducen en una desestabilización en el balance del poder regional o subcontinental, que finalmente puede $o$ no llevar al fracaso de la ola de democratización.

El impacto hegemónico, entonces representa un estiramiento en la ola de democratización que bajo condiciones no normales como lo es la intifada se conformaron Estados con regímenes híbridos (Morlino, 2008), autoritarismos competitivos (Levitsky \& Way, 2002) o Estados semisoberanos que optaron por la democracia en principio pero que por su propia naturaleza e historia no se han podido consolidar bajo este sistema político, rescatando pues que la democracia nunca fue un desarrollo empírico u obvio para los Estados modernos y parafraseando un poco a Mauricio Jaramillo Jassir (2018) la democracia es entonces un mal menor ya que este modelo ha dejado de ser un ideal por excelencia, pero según Morlino (2008) la legitimidad del régimen se da a partir de aceptación del aparato ideológico y una represión política por parte de las elites políticas y sociales que a su vez plantean un gobierno de transición que estabilice al Estado entre un modelo democrático y no democrático.

Históricamente los Estados desde su conformación reconocen la necesidad de consolidar un brazo armado, que en principio está bajo el mando del gobierno nacional, y es allí donde se consolida el poder militar que asegura su supervivencia. Ahora bien, desde el punto de vista de los racionalistas y a la luz de la teoría de la transición de poder, este no va a ser el único incentivo que sostenga una hegemonía 
en el sistema internacional, ya que, si este no cuenta con un poder material real, no va a tener éxito ni crecimiento en el sistema. En otras palabras, el interés de los Estados está orientado a mantener e incrementar la seguridad y la prosperidad en la mayor cantidad posible, y en especial si se comparte un lazo idiosincrático, como la democracia, ya que esta última sostiene que los Estados democráticos son menos propensos a caer en un conflicto. Eso permitiría que se encuentren un poco más protegidas ante el próximo impacto hegemónico que plantearía la ascendencia de una nueva potencia.

Morlino (2008), dentro de las tres categorías que plantea para clasificar a los Estados híbridos, categoriza a Marruecos en "Protected democracies" o democracias de dominio, citando un poco a Croissant y Merkel (2000), los cuales sostienen que la democracia está controlada por aparatos militares o incluso por fuerzas externas a las del país, que condiciona el régimen, o, en cualquier caso, que hay leyes o leyes no escritas que a su vez la limitan. Sin embargo, Morlino (2008) no encasilló a Túnez dentro de ninguna de las categorías que propuso, pero sí afirmó que Túnez se dirigía hacia un autoritarismo. No obstante, según el informe del Freedom House, para el año 2018 -en el cual se plantea una escala de 0 a 100 donde 0 es nada libre y 100 libre- Túnez, por su lado, era el Estado más sólido y democrático de la región, con un puntaje de 78 de 100, y guardando las proporciones se evidenciaba, a su vez, un protagonismo similar por parte de Marruecos, que obtuvo un puntaje de 41 de 100.

Sin embargo, desde la teoría se podría afirmar que al existir una transición hacia la democracia no se puede garantizar una reconfiguración en el balance de poder, ya que este se da a partir de la optimización y maximización de los recursos, que son al final los que refuerzan y sustentan el poder material real que tienen los Estados. El poder material que tienen los Estados son las herramientas para llevar a cabo, finalmente, la reconfiguración y estabilización en el 
sistema internacional. Ya que aquellos quienes tienen los recursos y determinan la oferta de estos son quienes instauran el crecimiento y la estabilidad de los demás Estados, es aquí donde es posible alinear estratégicamente los intereses geoeconómicos de los Estados con los intereses geopolíticos y proyectarlos en la política exterior que permita, a su vez, un desarrollo positivo en la obtención de los intereses nacionales y su ascendencia hegemónica.

Hudson (2005) analiza las estructuras que inciden en el policy making de EE. UU. en relación con Medio Oriente; a su vez, realiza un estudio de la "revolución neoconservadora" impulsada por George W. Bush desde su llegada a la presidencia en 2001, donde Bush es quien promueve una actitud más agresiva e intervencionista de Estados Unidos en el mundo árabe. A partir de la Segunda Guerra Mundial, EE. UU. toma relevancia en el sistema internacional y se instaura como hegemónico, postulando así su sistema político y económico como la mejor alternativa para los Estados, al tiempo que iniciaba una batalla ideológica y militar con la URSS, en cuyo caso los más perjudicados fueron las periferias debido a que en su momento sirvieron, y se puede afirmar que aún, sirvieron como escenarios de confrontación para las potencias. Pese a esto, al interior del mundo árabe se buscó crear una integración identitaria, bien conocida como el panarabismo, con el fin de fortalecer y proteger sus raíces ideológicas y culturales de occidente. En cuanto a sus recursos, especialmente los hidrocarburos, y luego de la conformación de los No Alineados en el periodo de Guerra Fría, se conformó la Organización de Países Exportadores de Petróleo (OPEP) en 1960, desde la cual los países de esta región crearon un contrapeso como defensa hacia los centros y sus dinámicas dominantes. Sin embargo, Marruecos y Túnez no son miembros de esta organización internacional, y luego de la Primavera árabe se dio una mayor occidentalización como anteriormente se ha mencionado. 
Por su lado, Izquierdo F. (2009), en la obra titulada Poder y regímenes en el mundo árabe contemporáneo, afirma que:

La inestabilidad en Oriente Medio y el Norte de África fue provocada principalmente por actores externos, a mediados del siglo XX, las independencias darían paso a la entrada en escena de nuevos actores. La creación de nuevos estados y la consolidación de la soberanía en los ya existentes desencadenaron la competición por el poder de las élites de cada uno de estos países. (Izquierdo, 2009, p 10)

Sumándole que no existe una transformación real producto de una revolución desde abajo, ya que solo se han logrado débiles reformas, las élites de la región no se encuentran en la disposición de ceder el poder, por lo cual truncan las olas de democratización manteniendo así las lógicas del statu quo. A su vez, Laura Feliu y María Angustias Parejo (2009), en su capítulo titulado "Marruecos: la reinvención de un sistema autoritario", sostiene que:

La evolución modernizadora del cambio de cultura política, de sectores que han accedido a una formación superior, de constreñimientos macroeconómicos y condicionantes derivados de la globalización sigue transformando la apariencia del Estado y de la sociedad, pero sin lograr transformar los pilares en los que se sustenta el poder. (Feliu y Parejo, 2009, p. 102)

Sin embargo, y pese a la estabilización política y social de la cual goza Marruecos, en la década de los 90, luego de los sucesivos golpes de Estado militares de los años setenta, cae nuevamente en una inestabilidad por los atentados de inspiración salafista a inicios del siglo xxi, los cuales muestran el peligro de un gobierno en solitario y absolutista, por cual Marruecos, en un primer momento, se ve en la obligación de ampliar su base política y, en segundo lugar, en buscar aliados estratégicos y formular herramientas que le permitan controlar y mitigar la ola de pobreza multidimensional que se vive al interior del país y en la región.

No obstante, los mecanismos y las herramientas propuestas no satisfacen las necesidades de la población y por el contrario en la 
región se vive un contagio en las demandas y necesidades de la población, así como una inconformidad constante por sus gobiernos absolutistas, la última manifestación de inconformidad colectiva con los gobiernos absolutistas se evidencio durante la intifada.

Todo esto provoca revueltas incontrolables, y una de las más conocidas e inspiradas en la historia europea del siglo XV al XIX fue la "revuelta del pan" o "motín del pan", que tenía como fin reducir los precios de este producto para hacerlo asequibles a la población civil que no pertenecía a la élite; sin embargo, en Túnez ya se había dado esta revuelta en 1987 al sumarse el crecimiento en el índice de pobreza multidimensional ajustado según la intensidad de las privaciones y el descontento con el gobierno, por el incumplimiento de la promesa de Ben Ali de permanecer en el poder únicamente dos períodos presidenciales, lo cual finalmente desemboca en el año 2011 en la "rebelión del jazmín" o revolución tunecina.

Pero en el caso de Marruecos, y pese a las inconformidades y demandas similares de la población, la monarquía se encontraba tranquila gracias a las ayudas, remesas procedentes de la emigración, acuerdos comerciales con Europa, etc.

Adicionalmente, se destacan dos fases de liberación: la primera, proviene de las presiones internas e internacionales; la segunda, viene dada por la causalidad de la apertura en el régimen y activando el campo político, permitiendo el acceso del islamismo político moderado a la arena política y electoral, lo cual permite la existencia y participación de la oposición.

Según Feliu y Parejo (2009), el nuevo autoritarismo se caracterizaría por dos procesos simultáneos: el primero es un reforzamiento y concentración en la cúspide autoritaria; el segundo es la ampliación en la base electoral. Ahora bien, la dimensión electoral se 
ha fortalecido mientras la represión sufre una reducción considerable desde 1993, posibilitando la participación de nuevos actores en el campo político; sin embargo, paralelamente se ha revitalizado la dimensión autoritaria.

El nuevo autoritarismo revitaliza la función de cooptación y de reparto de bienes privados que ya venían realizando las instituciones estatales convencionales y crea nuevas agencias que promueven la creación y el fortalecimiento de nuevos círculos élites que buscan obtener el poder político.

En la contemporaneidad se evidencia un repudio hacia los gobiernos que violan los derechos humanos constantemente, y más aún con los gobiernos del Machrek, que cometen esta clase de hechos en nombre de la tradición, el islam, la construcción nacional y el desarrollo; se evidencia incluso que son los privados e instituciones no tradicionales aquellos quienes tienen acceso a las materias primas y controlan el sector económico permeando el poder político y condicionan las políticas públicas.

Para apoyar la anterior afirmación, según una encuesta de la organización norteamericana Pew Research Center, desarrollada en el 2018, el nivel de rechazo del islam en países de Europa es de España (50 \%), Italia (69 \%), Hungría (72 \%), Francia (29 \%), Reino Unido $(28 \%)$ y Alemania (29\%). Gracias a lo anteriormente mencionado, es por lo que se otorgan insumos para la proliferación de los conflictos internos y se incrementan las posibilidades de fracaso para las olas democratizadoras. Pero desde la perspectiva de Kabunda Badi M. (2000) para lograr un cambio en el sistema y en la realidad de África se debe dar una transformación real de la historia tradicional y sus costumbres que son producto de una herencia colonial y una tradición inventada bajo prácticas etno-fascistas de las élites, además del forzamiento de integración étnico e islámico. 
Apoyando el argumento anterior, Kabunda y Mbuyi (2011), en colaboración con otros autores, buscan dar respuesta al fracaso de la cooperación e integración en África, y argumentan que esto se debe a la falta de financiación en lo social, también al hecho de no diagnosticar adecuadamente las causas profundas que existen al interior de los Estados. Pese a ello, abogan por el fortalecimiento de la cooperación sur-sur totalmente horizontal, como se mencionó al inicio de este artículo de investigación. Esta es una de las alternativas más viables para que los países africanos adquieran protagonismo en la geopolítica global y mayor margen de maniobra en el sistema internacional. Dejando de lado las teorías desarrollistas obsoletas y desde un enfoque racionalista, los países de África, Asia y América Latina, quienes son el tercer mundo, pero a su vez desde la geopolítica, constituyen el Heartland, por lo que son estas zonas aquellas que realmente cuentan con los medios y las capacidades materiales para transitar y posicionarse como los nuevos centros y hegemonías, transformando así la realidad internacional y dando vuelco a las estructuras de poder tradicionales.

\section{CONCLUSIONES:}

Por la naturaleza de los Estados africanos y de Medio Oriente, así como el desarrollo de su consolidación como regímenes híbridos, en el mundo árabe y Medio Oriente no es viable llevar a cabo una ola de democratización, aún si Estados como Marruecos y Túnez han optado por tener un acercamiento con occidente y su sistema político preponderante. Desde finales del siglo xx y en especial en el corrido del siglo xxi se han evidenciado falencias estructurales del modelo democrático; por otro lado, y rescatando un poco los postulados de Jaramillo (2018), la democracia es un mal necesario para los Estados en transición; sin embargo, sus dinámicas propias al ser diferentes terminan transformándose en un sistema híbrido que transita entre la democracia y la no democracia de manera pendular, lo cual deja 
en un limbo el andamiaje institucional doméstico y por lo cual los países vecinos sienten un especial rechazo. Las oscilaciones e inestabilidades políticas, junto con su debilidad orgánica doméstica son lo que finalmente termina en una polarización de la población, desembocando en el truncamiento en la cooperación regional y global.

Estos factores no permiten el éxito del incipiente sistema, por ende una reconfiguración a la jerarquía impuesta globalmente por las potencias tradicionales y emergentes que promueven el desarrollo de una "paz negativa" sometida a los intereses económicos de instituciones no nacionales e incluso no convencionales que buscan el mantenimiento del statu quo y por ende una cesación en la movilidad social, mediante el desarrollo de mecanismos y políticas públicas que beneficia considerablemente a las potencias tradicionales y mantiene una lógica colonial y de sometimiento a los países en vías de desarrollo.

Por otro lado, si se busca obtener el éxito en la reconfiguración del sistema, es necesario plantear estrategias alineadas con los intereses nacionales geoestratégicos y geopolíticos que mitiguen el choque de intereses con las potencias -se estaría hablando propositivamente de soberanía, autonomía o del principio de no intervención-, unificando a la población al interior de los Estados y su ordenamiento doméstico, por lo que los Estados deben formular proyectos de fortalecimiento institucional que permitan una atenuación en los conflictos internos y de administración tanto política como judicial; sin embargo, se debe tener cuidado de no caer en caudillismos, populismos e incluso en una mercadería política.

Al hacer esto se solidifica un poco la institucionalidad y plantea un escenario óptimo para la captación de inversionistas y aliados estratégicos de cooperación que le brinden herramientas y capacidades que permitan un crecimiento económico e industrial, todo esto, entrelazado a un enriquecimiento político y de desarrollo humano. 
Si el Estado logra mantener un equilibrio en su transición mediante estrategias de planeación a mediano y largo plazo de lo público, puede abandonar sus dinámicas de dependencia y auto-marginalización periférica, a la cual están sometidos los países africanos.

\section{ANEXO}

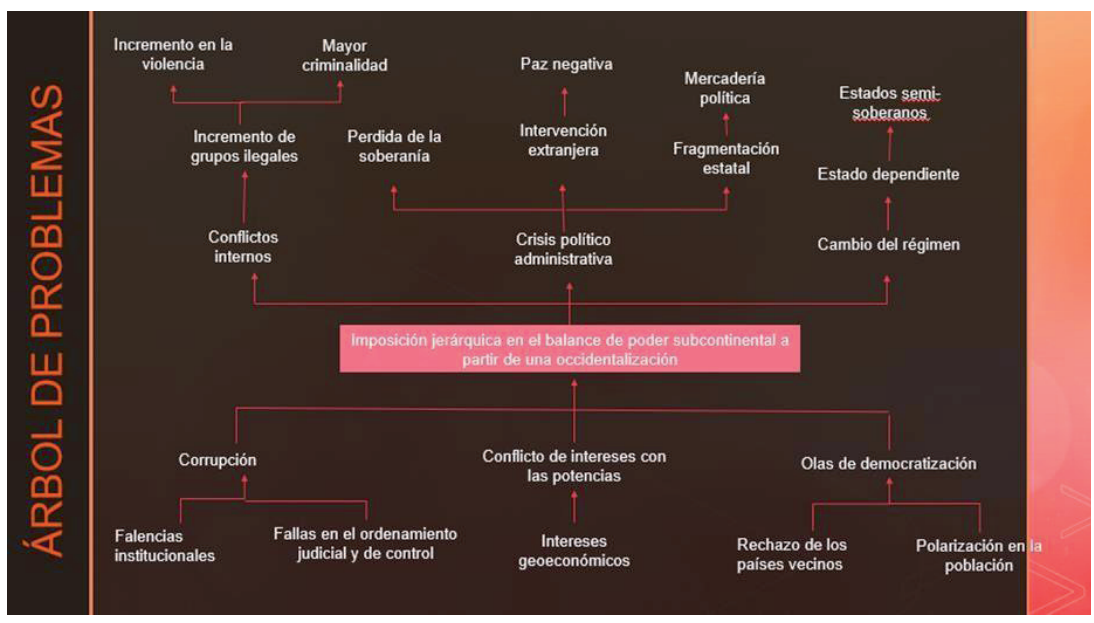

(elaboración propia, 2020)

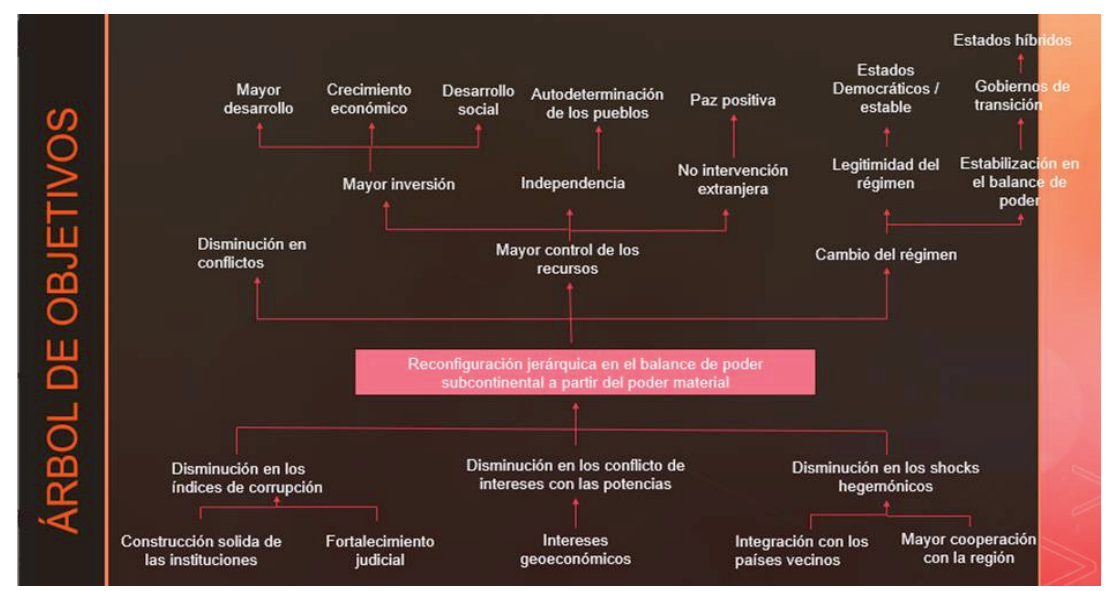

(elaboración propia, 2020) 


\section{LISTA DE REFERENCIAS:}

Altable, J. (2000). Oriente próximo: las claves del conflicto. Madrid, España: Silex Ediciones.

Bernal, L. (2018). Africanía: la evolución de las relaciones internacionales Iberoamérica-África subsahariana a la luz de las teorías de la periferia. En C. Ross y M. Badi Kubunda (eds.), Tránsitos materiales e inmateriales entre África, Latinoamérica y el Caribe (pp. 15-25). Santiago: Ariadna Ediciones.

García, J. (2002). La crisis de Marruecos. Estudios de Política Exterior S.A.

Freedom House. (2019). Democracia en retirada: La libertad en el mundo 2019. Washington, USA: Freedom House.

Grovogui, S. N. (2011). La soberanía en África: casi estados y otros mitos en la teoría internacional. Relaciones internacionales, (18), 137-155. Recuperado de https://revistas. uam.es/relacionesinternacionales/article/view/5100

Gunitsky, S. (2017). Aftershocks: Great Powers and Domestic Reforms in the Twentieth Century. USA: Princeton University Press.

Hudson, M. C. (2005). The United States in the Middle East. En L. Fawcett, International Relations of the Middle East (pp 283-305). New York, USA. Oxford University Press.

ICEX y Gobierno de España. (2019). Ficha País de Túnez. Madrid, España: Secretaría del gobierno de España.

ICEX y Gobierno de España. (2019). Ficha País de Marruecos. Madrid, España: Secretaría del gobierno de España.

Izquierdo, F. (Ed.) (2009). Poder y regímenes en el mundo árabe contemporáneo. Barcelona: CIDOB.

Jaramillo Jassir, M. (2018). La vigencia de Sartori en las inacabadas definiciones de democracia y régimen democrático. DESAFIOS, 30(1), 359-366.

Kabunda Badi, M. (2000). Derechos humanos en África: Teorías y prácticas. Bilbao: Universidad de Deusto.

Kabunda, Mbuyi (coord.). (2011). África y la Cooperación con el Sur desde el sur. Madrid: Catarata. 
Levitsky, S., y Way, L. (2002). The rise of competitive authoritarianism. Journal of Democracy, 13(2), 51-65. Recuperado de https://scholar.harvard.edu/levitsky/files/SL_elections.pdf

Morlino, L. (2008). Hybrid Regimes or Regimes in transition? Madrid: Fundación de Relaciones Internacionales y el diálogo exterior (FRIDE).

Navarro Palazón, J., y Trillo San José, C. (2018). Almunias. Las fincas de las élites en el Occidente islámico: poder, solaz y producción. Madrid: CSIC.

Neila Hernández, J. (2011). España y el Mediterráneo en el siglo XX: De los acuerdos de Cartagena al proceso de Barcelona. Madrid: Sílex Ediciones.

Reynoso Brito, P. (2019). La teoría del Cambio Sistémico y el desarrollo militar-espacial de la República Popular China [Trabajo de grado]. Universidad de las Américas Puebla, México.

Rocha Valencia, A., y Morales Ruvalcaba, D. (2018). El poder nacional-internacional de los Estados. Una propuesta trans-estructural. Geopolítica(s). Revista de estudios sobre espacio y poder, 9(1), 137-169

Rostow, W. W. (1961). Las etapas del crecimiento económico. Fondo de Cultura Económica, México

Tariq, A. (2002). El Choque de Los Fundamentalismos: Cruzadas, Yihads y Modernidad. Madrid: Alianza Editorial S.A.

Youssef Bouajaj (2018). Islam y democracia: el caso de Indonesia. MENAnalisis. Recuperado de http://www.menanalisis.com/islam-y-democracia-el-caso-de-indonesia 